\section{Nur unterstützender Beitrag}

\section{Das Angebot der "Service-Leisłungen" rund um das Öko-Audit wächst stetig. Vor allem im Softwarebereich werden laufend neve Angebote auf den Markt gebracht. Was sind die Einsatzmöglichkeiten von EDV im Rahmen eines Öko- Audits? Welche Anforderungen sind zu stellen und inwieweit werden diese heute erfült?}

$\mathrm{G}$

Von Beate Haas und Heike Köckler einem Berg an neuen Verordnungen herum, sondern sind auch auf der Suche nach geeigneter Software. Denn die komplexe Aufgabe, die mit der Umsetzung der Öko-Audit-Verordnung auf den Betrieb zukommt, läßt sich mit ihrer Hilfe wesentlich erleichtern. Allerdings gibt es bislang noch keine Untersuchungen, was eine hilfreiche Software beinhalten sollte und wobei sie unterstützend wirken kann. Welche Möglichkeiten gibt es also, Software bei der Umsetzung des Audits erfolgreich einzusetzen? Welche Anforderungen muß eine solche Software erfuillen? Welche dieser Anforderungen sind für einen bestimmten Betrieb als Notwendigkeit und Voraussetzungen anzusehen? Welche grundlegenden Voraussetzungen an EDV-Systeme sind im speziellen Fall für den Betrieb notwendig? Grundsätzlich lassen sich drei Typen von Software-Produkten in diesem Bereich ausmachen:

1. Einführungs-Programme, die vor allem an jene Betriebe gerichtet sind, welche vor der Entscheidung stehen, ob sie ein Öko-Audit durchführen sollen und wollen.

2. Prozeßbilanz-Programme, die der Ermittlung und Darstellung von Input- und Outputgrößen dienen und somit den Produktionsprozeß betrachten.

3. Managementsystem-Software, die mittels eines Fragenpools bei der Organisation und dem Aufbau eines Umweltmanagementsystems helfen.

Für diese drei Softwares wurden im Rahmen einer Arbeit an der Fakultät Raumplanung der Universität Dortmund Anforderungen formuliert, die eine Hilfe darstellen sollen, die Flut von Software-Produkten einzuordnen. Was sollen also beispielsweise Prozeßbilanz-Programme erfïllen?

\section{$>$ Anforderungen an ProzeBbilanz-Programme}

- Ein Prozeßbilanz-Programm soll Schnittstellen $\mathrm{zu}$ anderen Anwenderprogrammen bieten, damit ein Betrieb schon eingesetzte und verfügbare Daten, beispielsweise Produktbilanzen, weiterhin nutzen kann und die Neueingabe bereits erfaßter Daten vermieden werden kann. - Ein guter Aufbau sowie die Aktualisierung und Darstellung von Stoff- und Energieflüssen (eventuell in Kooperation mit anderen Anwenderprogrammen) ist von zentraler Bedeutung.

- Anschauliche Darstellungsmöglichkeiten (z.B. in Form von Diagrammen), insbesondere für die am Ende des Audits zu verfassende Umwelterklärung sollten ein elementarer Bestandteil des Programms sein.

- Verschiedene Prozeßvarianten als Hilfe in Abwägungsprozessen, beispielsweise unterschiedlicher Produktionswege sollten herausgebildet werden können.

- Umweltkennzahlen einzelner Branchen und Produkte innerhalb des Programms wären von Vorteil. Sie drücken die Umweltinanspruchnahme des jeweiligen Produktes in Relation aus, zum Beispiel den Energieeinsatz pro Produkt bei der Herstellung. Ihre Anwendung erleichtert daher die Vergleichbarkeit unterschiedlicher Produkte oder Produktbestandteile.

- Stehen verschiedene Produktionswege zur Abwägung, so hilft ein Bewertungsschema, welches die Auswirkungen einer bestimmten Produktion auf die Umwelt einstuft. Wenn diese Schemata flexibel gestaltbar sind, kann ein Betrieb durch das Festlegen von Richtwerten (wobei die Grundlagen der Richtwerte freigestellt sind) seine eigenen Leitlinien bezïglich der Umweltschutzmaßnahmen entwickeln. Dabei sollte die Nachvollziehbarkeit selbstverständlicher Bestandteil sein.

- Rekursive Netzwerke sollen zur Erleichterung der Wahl des ökologisch sinnvollsten und unternehmensstrategisch günstigsten Produktionsprozesses dienen. Rekursive Netzwerke ermöglichen es, von einem im Herstellungsprozeß entstandenen Schadstoff auf die entscheidenden Elemente der Produktzusammensetzung zu schließen. So lassen sich Rückschlüsse ziehen, in welcher Zusammensetzung die Ausgangsstoffe vorliegen sollten, damit die Belastung möglichst gering bleibt.

- Und nicht zuletzt sollten entsprechende Systeme zur Berechnung der Output-Zahlen integriert sein. Nur ein Vergleich des theoretischen und des realen Outputs kann beispielsweise zur Ermittlung von Leckagen etc. führen.

\section{Ergebnisse}

Es ist eindeutig eine große Produktpalette bei Anbietern von Prozeßbilanzierungs-Programmen zu finden, während das Angebot in den beiden anderen Bereichen weit geringer ist. Im Rahmen der Arbeit wurden explizit drei derzeit auf dem Markt gängige Programme untersucht, die bereits sehr umfassend sind und insbesondere den oben genannten Einsatzbereichen zugeordnet werden können. Die Softwareauswahl orientierte sich am Stand der Entwicklung, Juli 1996. Dabei hat sich gezeigt, daß die angebotenen Produkte sowohl in den drei Untersuchungsbereichen als auch innerhalb dieser sehr unterschiedlich ausgeprägt sind. Für ein Unternehmen ist es notwendig die zur Zeit als Insellösungen arbeitenden Programme miteinander zu verknüpfen. Wünschenswert wäre natïrlich eine Software, die den gesamten Ablauf des Öko-Audits - von der Prozeßbilanz über Managementsystem bis hin zur Umwelterklärung - abdeckt. Die Arbeit hat aber auch insgesamt bestätigt, daß bei aller Qualität der Software trotzdem klar ist, daß diese nur unterstïtzend zum Öko-Audit beitragen kann. Die betriebsinterne Auseinandersetzung mit der für viele Unternehmen neuen umweltbezogenen Denkweise kann Software nicht ersetzen, sondern nur befördern und den Umgang mit dieser Thematik erleichtern.

\section{Die Autorinnen}

Beate Haas und Heike Köckler sind beide Studentinnen an der Fakultät Raumplanung der Universität Dortmund.

Kontakte: Beate Haas, Krückenweg 8, 44227 Dortmund und Heike Köckler, Benrather SchloBallee 17c, 40597 Düsseldorf 
(c) 20I0 Authors; licensee IÖW and oekom verlag. This is an article distributed under the terms of the Creative Commons Attribution Non-Commercial No Derivates License (http://creativecommons.org/licenses/by-nc-nd/3.o/), which permits unrestricted use, distribution, and reproduction in any medium, provided the original work is properly cited. 